\title{
A Computational Model for the Overall Pattern of Ocular Dominance
}

\author{
David G. Jones, ' Richard C. Van Sluyters, ${ }^{2}$ and Kathryn M. Murphy ${ }^{3}$ \\ 1Department of Computer Science, Stanford University, Stanford, California 94305, 2School of Optometry, University of \\ California, Berkeley, California 94720, and ${ }^{3}$ Department of Psychology, McGill University, Montréal, Québec, \\ Canada H3A 1B1
}

\begin{abstract}
In layer IV of the primary visual cortex, in both the macaque monkey and the cat, geniculocortical terminals representing the two eyes are segregated into alternating zones known as ocular dominance bands. Viewed tangentially, in the monkey these bands take the form of a series of branching parallel stripes that run roughly perpendicular to the border of striate cortex. In the cat, the overall ocular dominance pattern consists of irregularly branching, beaded bands that exhibit no predominant orientation. If the striking differences in the appearance of these two patterns reflect important differences in the basic rules governing cortical ocular dominance, then this poses a problem for attempts to formulate general principles of visual cortical organization. However, it has been suggested that the differences in the appearance of the ocular dominance patterns in these two species could result simply from known differences in the boundary conditions of their geniculocortical pathways. This article describes the formulation and testing of a single computational model that accurately predicts the quite dissimilar ocular dominance patterns in cats and monkeys. This model also generalizes to predict the different ocular dominance patterns observed in young and old three-eyed frogs, supporting the notion that the overall pattern of ocular dominance is governed by a common set of rules. The significance of these results is discussed in terms of previous models, which have focused largely on local processes underlying the development of ocular dominance segregation. Although the present model is not a developmental one, it does shed some light on potential mechanisms for establishing retinotopy in striate cortex and on possible developmental relationships between the geniculostriate pathway and intrinsic modularity of the striate cortex.
\end{abstract}

In the primary visual cortex of cat and monkey, inputs from the two eyes, carried by geniculocortical afferents, are segregated into alternating left- and right-eye zones in layer IV. These form

\footnotetext{
Received Feb. 28, 1991; revised June 4, 1991; accepted June 27, 1991.

We are grateful to Dr. Martha Constantine-Paton for generously providing photographs and tissue of her three-eyed frog preparation, and we thank John Fiorillo for skilled photographic assistance. This work was supported by research grants to R.C.V.S. from the National Institutes of Health, National Eye Institute (EY02193), and the National Science Foundation (BNS-8418738), and by CORE Facilities Support Grant EY03176 from the National Eye Institute. K.M.M. is an NSERC University Research Fellow, and this work was supported in part by a research grant from the Natural Science and Engineering Research Council (URF$0045835)$.

Correspondence should be addressed to David G. Jones, Department of Electrical Engineering, McGill University, 3480 University Street, Montréal, Québec, Canada H3A 2A7.

Copyright $\odot 1991$ Society for Neuroscience $0270-6474 / 91 / 113794-15 \$ 05.00 / 0$
}

the anatomical basis for physiologically defined ocular dominance columns (Hubel and Wiesel, 1965, 1968). During development, the initially overlapping inputs from the two eyes are refined and become segregated into ocular dominance columns (LeVay et al., 1978, 1980). It is this process of segregation that has attracted much of the attention of previous computational models of ocular dominance. Several models have been proposed to account for how initially overlapping geniculocortical projections might segregate into eye-specific zones (von der Malsburg, 1979; Swindale, 1980; Miller et al., 1989). These models have been based on local interactions that lead to the segregation of inputs and in some cases have incorporated known local physiological and anatomical information into a comprehensive developmental model (e.g., Miller et al., 1989). However, even these increasingly sophisticated models of the segregation process, which accurately mimic the local appearance of ocular dominance, have remained unable to predict its overall pattern. It is also unclear how and why the parameters of these models must be modified for them to apply to the ocular dominance patterns found in other species.

The primary visual pathways of the cat and monkey share many similarities. For both of these highly binocular animals, retinal ganglion cells project from the two eyes to retinotopic maps in separate layers of the dorsal lateral geniculate nucleus (LGN), which then project to layer IV in the striate cortex, but in segregated regions. These eye-specific regions, or ocular dominance columns, have, for each species, a characteristic size, spacing, and overall pattern. Only within the extragranular layers of the cortex are the signals from the two eyes combined extensively. With such similarities in their early visual pathways, it is perplexing then to see that the overall, two-dimensional (2-D) patterns of ocular dominance are quite dissimilar in these species (Fig. 1).

When viewed tangentially, in the monkey, ocular dominance columns take the form of a series of branching parallel stripes that many have likened to the pattern of stripes on a zebra (Fig. $1 A$ ). These stripes tend to run roughly perpendicular to the border between cortical areas 17 and 18. In the cat (Fig. 1B), the ocular dominance pattern differs from that in the monkey, consisting of irregularly branching, beaded bands that exhibit no general tendency to be oriented orthogonal to the $17 / 18$ border (Anderson et al., 1988). Superficially at least, these observations leave open the possibility that ocular dominance patterns in these two species could be organized according to different rules, reflecting, perhaps, different underlying functional organizations. Instead, it has been suggested that the monkey ocular dominance pattern could result from the boundary conditions of the geniculocortical projection (LeVay et al., 1985), 
and that a similar argument may apply to the overall pattern of ocular dominance in the cat (Anderson et al., 1988).

Given that the inputs from the two eyes will be segregated, LeVay et al. (1985) have provided an informal argument for why one might expect ocular dominance domains to be in the form of bands running roughly perpendicular to the long axis of primary visual cortex. Their argument is based on the anatomical observation that, in the macaque, the largest LGN layers are roughly circular (Connolly and Van Essen, 1984), whereas primary visual cortex is approximately twice as long as it is wide. Thus, to a first approximation, the geniculocortical projection maps two circular disks in the LGN (one from each eye), into a 2:1 ellipse in the cortex. One intuitively simple way to achieve this mapping would be to slice each LGN disk into vertical strips and then to interdigitate the left-eye and righteye strips to fit snugly into the elongated cortical ellipse (Fig. 2). In fact, LeVay et al. suggest this is the only way to obtain elongation along one axis without anisotropic stretching or compression of the LGN inputs to the cortex.

A similar informal argument has been put forward to account for the 2-D pattern of ocular dominance observed in cat visual cortex (Anderson et al., 1988). As these authors pointed out, in the cat, the LGN layers and striate cortex have similar, oval shapes (Fig. 2). As a result, there is no single axis along which the cortical map may be elongated by interdigitating stripes of LGN input, unless the LGN inputs are compressed in one direction (but see Appendix). Anderson et al. (1988) proposed that the geniculocortical projection is subject to the constraint of mapping the two representations of the visual hemifield into area 17 , as well as to the constraint of minimizing the amount of anisotropic stretching or compression of the retinotopic maps within the projections for each eye. This suggests that there should be no overall tendency for ocular dominance bands to adopt a particular orientation in cat striate cortex; indeed, there should be a uniformly random distribution of ocular dominance band orientations.

This article describes our efforts to build on these intuitive ideas and formulate a single computational model that accurately predicts the quite dissimilar ocular dominance patterns of cats and monkeys. We propose that the overall pattern of ocular dominance is dictated by a set of relatively simple rules that govern the geniculocortical projection. First, it is assumed that the 2-D shapes of the LGN layers and primary visual cortex are predetermined, along with the polarity of their retinotopic maps. Second, since it is the overall pattern that is of primary interest, the segregation and characteristic width of ocular dominance columns are taken as a given. Third, it is argued that the proportion of the cortical visual map devoted to the representation of the central visual field is unchanged from that in the LGN and that the visual map within individual ocular dominance columns is isotropic. Fourth, the ubiquitousness of retinotopic maps throughout the early visual pathway is incorporated in our model as a constraint that neighboring points in the visual field will have nearby cortical representations. Finally, we propose a simple metric for characterizing how well a candidate geniculocortical mapping satisfies these constraints, thus allowing a determination of which map is the best. All of these considerations are justified biologically, but they are also essential in making the model precise enough to give unambiguous predictions and thus be testable.

From these assumptions about the shapes of the LGN and cortex, and the polarities of the retinotopic maps, it is possible to determine the cortical ocular dominance patterns predicted by our model. For both the cat and monkey, the patterns predicted by our model bear a strong similarity to those actually observed. This suggests that in both cats and monkeys, the overall pattern of ocular dominance is a simple consequence of identical constraints that at once provide segregated visual inputs, while keeping representation of neighboring points nearby, subject literally to the boundary conditions present in each species.

In the following sections, we describe in a more formal way the development of the model, how it was implemented computationally, the results of testing our model with this implementation, and the significance of these results for cortical architecture.

\section{Development of the Model}

Despite the differences in the overall patterns of ocular dominance found in the cat and monkey, we claim that these patterns are the consequence of a common set of rules that govern the geniculocortical projection. In order for this notion to be tested, this single set of rules must be explicitly stated as a model. Before giving a concise statement of our model, however, the evidence underlying some of the points needs to be elaborated. The following describes the four sets of observations and assumptions that form the basis of our ocular dominance model.

\section{2-D shapes of the $L G N$ and striate cortex}

The basic intuitive idea behind the present model is one based on shape. While it might seem more appropriate to start from the retina (cf. Anderson et al., 1988), this poses a difficulty when considering shape. The difficulty lies in the fact that the density of cones or retinal ganglion cells is higher in the central retina and progressively lower toward the periphery. Given this complication, and the fact that our interest lies primarily in the pattern of visual input to the cortex, we have limited our focus to the projection from the layers of the LGN to striate cortex (but see Discussion).

The same location in visual space is represented throughout the thickness of the LGN layers, while parallel to their surface there is a precise visuotopic map, with the largest layers of the LGN containing a complete map of the contralateral visual hemifield. It is the (unfolded) 2-D shape of these layers that is of importance for the present model.

In the monkey (top left of Fig. 2), the larger LGN layers are roughly circular, with the foveal representation lying at the dorsal pole and the vertical meridian running around most of the circumference (Connolly and Van Essen, 1984; LeVay et al., 1985). In the cat (top right of Fig. 2), the LGN layers are roughly 2:1 ellipses, with the representation of the area centralis lying near the center of the medial (long) edge and the vertical meridian extending along that edge (Sanderson, 1971). In both cases, since the maps are retinotopic, the horizontal meridian is perpendicular to the vertical meridian at the foveal representation and runs through the center of the LGN to the opposite, edge.

In the monkey (bottom left of Fig. 2), when the primary visual cortex is unfolded, it takes the form of an ellipse that is longer than it is wide by slightly more than a factor of 2 (LeVay et al., 1985). The foveal representation lies at one end of this ellipse, adjacent to extrastriate area 18. Wrapping around this narrow end of the ellipse, the representation of the vertical meridian extends along the border between areas 17 and 18 (LeVay et 
A
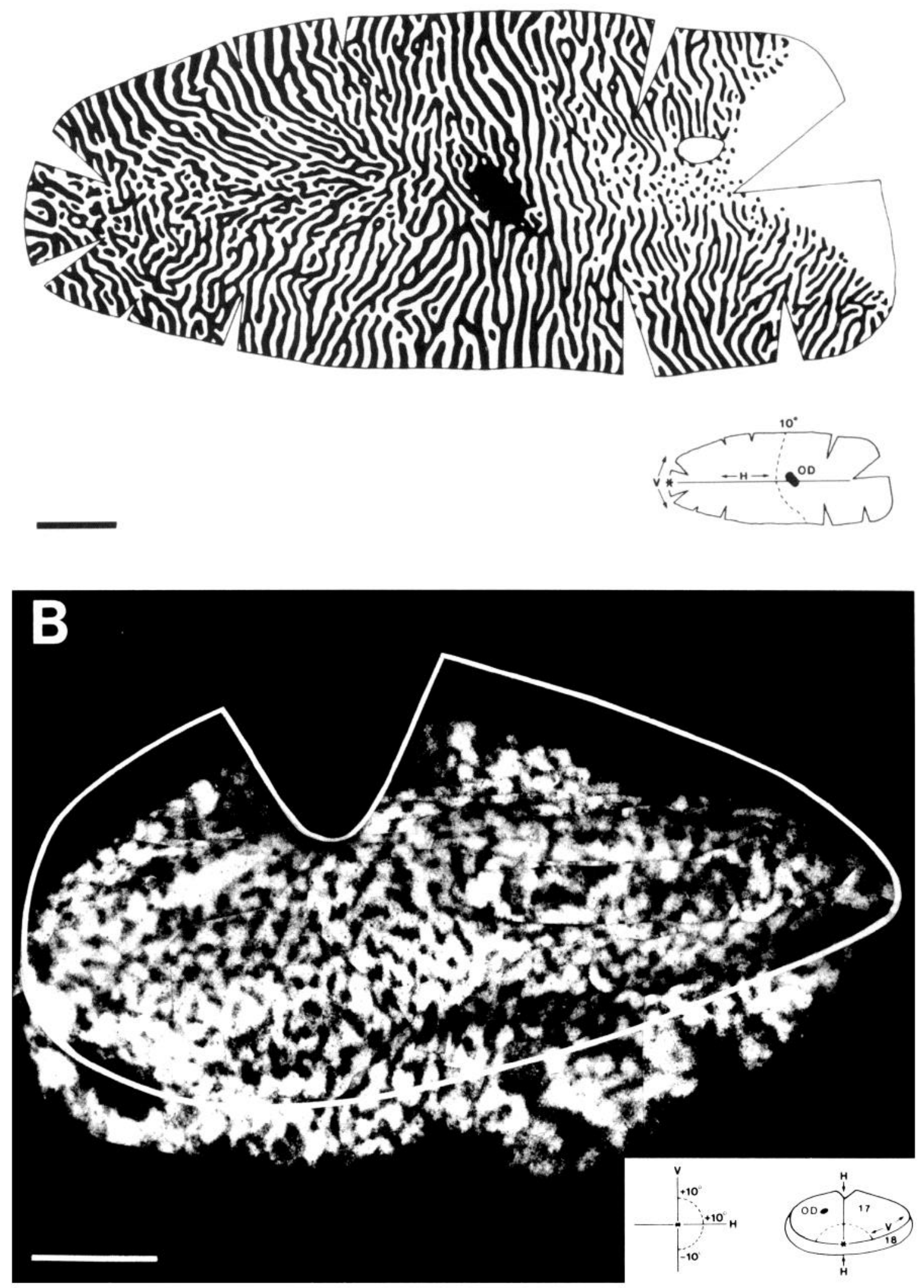


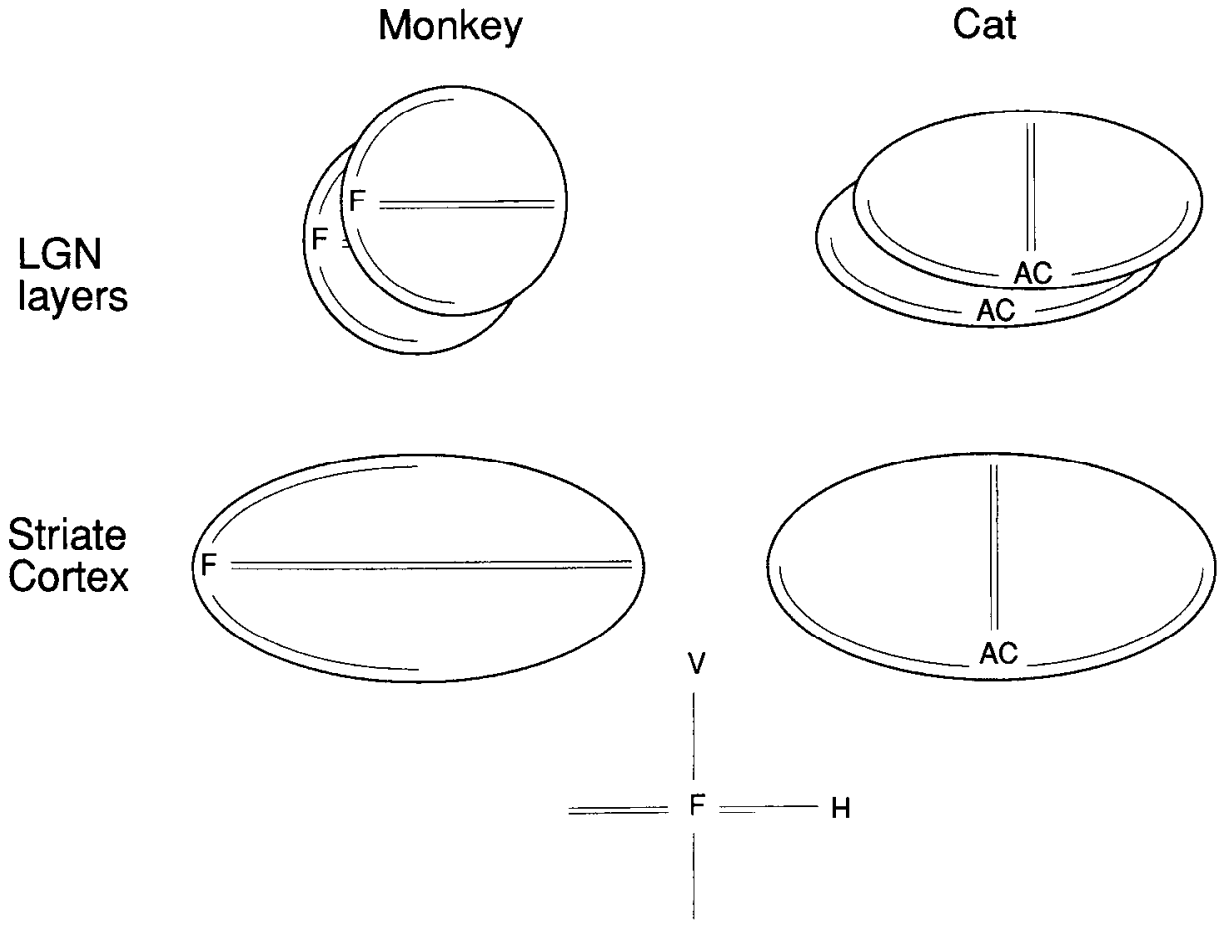

Figure 2. Retinotopic maps and shapes of the LGN layers and primary visual cortex. This schematic diagram shows the 2-D shapes of representative right- and left-eye LGN layers and the striate cortex in the monkey (left) and cat (right), with key retinotopic landmarks indicated, including the representation of the fovea $(F)$ or area centralis $(A C)$, the vertical meridian (single line), and the horizontal meridian (double line). LGN layers in the monkey are roughly circular, while those in the cat are elliptical. Although striate cortex is elliptical in both species, in the monkey, the foveal representation is located at one pole, whereas in the cat, the area centralis representation is found at the center of one long side. al., 1985). In the cat (bottom right of Fig. 2), the 2-D shape of flattened primary visual cortex is also an ellipse, with a lengthto-width ratio of slightly more than 2:1 (Anderson et al., 1988). The representation of the vertical meridian extends along the dorsolateral edge of this ellipse separating areas 17 and 18 , but in the cat, this is the long edge of the ellipse. The representation of the area centralis lies at the center of this long edge (Tusa et al., 1978).

\section{Proportional projection}

The intuitive argument based on the shapes of the LGN and striate cortex depends implicitly upon the fact that the geniculocortical projection is uniform (Clark, 1941). Virtually all retinal ganglion cells project to the LGN (Bunt et al., 1975) and from there are relayed to striate cortex. However, it has long been contentious whether the initial sampling of the visual field is maintained through successive stages of the visual pathway, or whether the central visual field, which is sampled more densely in the retina, is even further emphasized by devoting disproportionately more geniculate and cortical neurons to the representation of the central visual field. A number of recent studies support the conclusion that there is no selective magnification and that instead the numbers of LGN cells and cortical cells allocated for the representation of a region of the visual field are proportional to the number of retinal ganglion cells in both the cat (Stone, 1965; Tusa et al., 1978) and monkey (Schein and de Monasterio, 1987; Schein, 1988; Wässle et al., 1989, 1990).
From the standpoint of the present model, the assumption taken from this recent work is that a given area of an LGN layer projects to a roughly proportional area of the cortex, regardless of position within the map of the visual field. However, a small gradient of cortical magnification across the striate cortex would have little effect on the predictions made by our model.

\section{Isotropy in the cortical visual map}

The cortical magnification factor is defined as the number of millimeters of cortex per degree of visual field (Daniel and Whitteredge, 1961). Such a measure could equally well be applied to the LGN layers. We may ask whether these visuotopic maps are locally isotopic, with the linear magnification factor independent of the direction of measurement, or whether they are anisotropic, reflecting an expansion or compression in some direction.

In the cortex, this question is further complicated since the answer may be different depending on whether we restrict our attention to within individual ocular dominance columns, across several neighboring ocular dominance columns, or across the entire striate cortex. In the granular layers, visual space is doubly represented because it is carried separately by the projections from the right and left eye. In the extragranular layers, there is a composite, binocular visual map. Consider this composite map in a region of the cortex where ocular dominance columns take the form of alternating parallel bands (Fig. 3). In the di-

Figure 1. Overall pattern of ocular dominance. $A$, Drawing of the overall tangential pattern of cortical ocular dominance columns revealed in macaque primary visual cortex by autoradiography following injection of $\mathbf{H}^{3}$-proline into the contralateral eye (from LeVay et al., 1985 ). $B$, Photographic montage of the overall ocular dominance pattern in a cat. Lighter areas show the visual inputs labeled in the striate cortex ipsilateral to an eye injected with WGA-HRP (from Anderson et al., 1988). Small insets indicate the locations of the primary visual cortex or area 17 (17) and the adjacent cortical visual area $18(18)$, as well as the approximate locations of key retinotopic landmarks, including the cortical representation of the optic disk $(O D)$, the fovea or area centralis $(*)$, the horizontal $(H)$ and vertical $(V)$ meridians, and the central $10^{\circ}$ of the visual field. Scale bars, $5 \mathrm{~mm}$. 


\section{LGN}

Layers

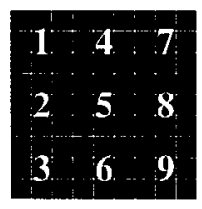

Ipsi

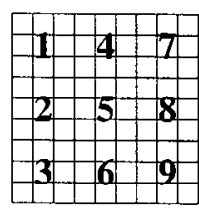

Contra
Ocular Dominance

Columns
Composite

Visual Map

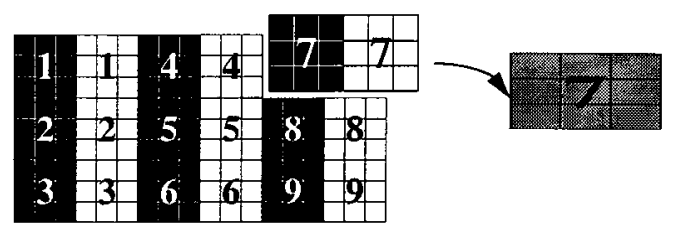

Locally Isotropic

Globally Anisotropic

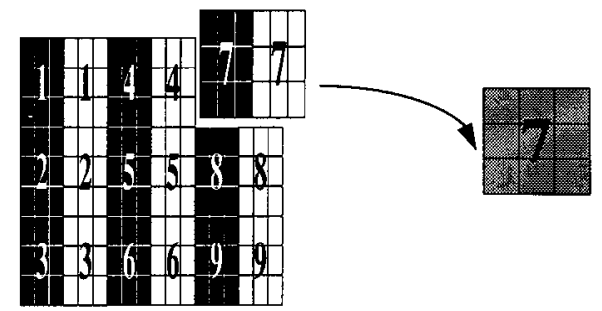

Locally Anisotropic

Figure 3. Comparison of local and global isotropy. Diagrams on the left represent the continuous retinotopic maps formed within separate LGN layers by inputs from the ipsilateral (black) and contralateral (white) eyes. Diagrams in the center and on the right represent the visual maps present in the granular and extragranular layers of striate cortex, respectively. At the level of the granular layer, the maps from the ipsilateral and contralateral eyes remain separate and take the form of interdigitating ocular dominance columns. In the extragranular layers, inputs from the two eyes are combined into a single, composite, binocular visual map. A map is said to be isotropic if the cortical magnification factor is the same regardless of the direction in which it is measured. When inputs from the two eyes interdigitate to form orderly parallel stripes, it is impossible for both the local (within ocular dominance column) and global (across many ocular dominance columns) visual maps to be isotropic. If the map within each ocular dominance column is isotropic, then the composite visual map must be anisotropic (top right). For the composite visual map to be isotropic, the map within each ocular dominance column would need to be compressed in one direction (bottom right).

rection parallel to these bands, the composite map shares the same magnification factor present within the individual ocular dominance columns. Perpendicular to these bands, however, the composite map traverses the visual field half as quickly as within any individual ocular dominance column. Thus, if the map within each column is isotropic, then across several columns the composite binocular map is necessarily anisotropic by a factor of 2 (Hubel and Wiesel, 1977; Connolly and Van Essen, 1984). Conversely, an isotropic composite map would require the map within ocular dominance bands to be anisotropic. When the ocular dominance columns form parallel bands, the within-column map and the composite map cannot both be isotropic (Fig. 3).

Working backward from an assumption that the composite binocular visual map in the monkey was isotropic, Hubel and Wiesel (1977) reasoned as above that the monocular visual map in layer IV must be anisotropic, with the map compressed along the horizontal meridian. A clearer and more direct answer to the question of isotropy lies in anatomical evidence from 2-deoxyglucose studies of the retinotopic map across macaque visual cortex (Tootell et al., 1988). Cortical magnification was measured both parallel and perpendicular to ocular dominance bands, and the results were in accord with the ratio of withincolumn cortical magnification factors in these two directions being $1: 1$. Thus, in this species, cortical magnification within a single ocular dominance column appears to be isotropic. This conclusion is further supported by the observation that the local anisotropies in the composite cortical map are related to the direction taken by the ocular dominance bands, with the com- posite map being more markedly anisotropic wherever ocular dominance bands form more orderly parallel stripes (Schwartz, 1985; Tootell et al., 1985).

Since, for the most part, ocular dominance bands in the monkey run perpendicular to the long axis of the elliptical striate cortex, this local, within-column isotropy introduces an overall 2:1 anisotropy in the composite binocular visual map. To our knowledge, there is no direct evidence on within-column isotropy in the cat, but even if it is 1:1 like the monkey, the random assortment of ocular dominance band orientations means that any local anisotropies introduced by side-by-side isotropic ocular dominance bands would tend to be cancelled out over the composite visual map as a whole.

The assumption of local isotropy is important to our model because it means that on the scale of individual ocular dominance columns, the visuotopic map cannot be compressed or distorted to fit into the target cortical map's shape.

\section{Neighborhoods}

Primary sensory areas in the cortex contain precise maps that maintain the neighborhood relationships of the sensory receptors, whether they be somatotopic, tonotopic, or retinotopic. It has been proposed that sensory areas, and visual areas in particular, contain topological representations because the intracortical horizontal connections required for lateral interactions in early sensory processing are kept short, and furthermore, these connections are developmentally much easier to specify (Cowey, 1981). In the case of vision, building up more elaborate receptive-field properties such as orientation or stereo disparity tun- 
ing using inputs from neighboring positions in the visual field becomes more economical as neighbors are represented closer together in the cortex. In fact, there is good evidence that the vast majority of connections within a visual arca are local and involved in receptive-field tuning (Cowey, 1979). The goal of having neighboring points in visual space represented at nearby locations in the primary visual cortex will turn out to be the driving constraint in our model.

\section{Statement of the model}

Based upon the observations outlined above, we may now concisely state our model governing the overall pattern of ocular dominance.

(1) The 2-D shapes of the LGN layers and primary visual cortex are predetermined, as is the location of the foveal representation $(\mathbf{F})$.

(2) That ocular dominance bands arise and taken on a characteristic spacing is taken as a given. It is their overall pattern that this model addresses.

(3) The geniculocortical projection is proportional and isotropic within individual ocular dominance bands.

(4) Points (1)-(3) above allow the relevant aspects of the LGN layers and primary visual cortex to be adequately modeled by a discrete collection of points lying within 2-D boundaries of the appropriate shape.

(5) The geniculocortical projection maintains neighborhood relationships by mapping adjacent LGN points, as well as corresponding points from the two eyes, to nearby locations in striate cortex.

(6) Candidate cortical maps can be evaluated in terms of the degree to which they satisfy this last constraint by measuring the maximum distance between cortical representations of neighboring LGN points (D), and the number of pairs of points that are this far apart (N). Of all possible maps, those that minimize $\mathbf{D}$ and $\mathbf{N}$ best satisfy the above constraints, and so may be called optimal.

\section{Method}

Our model defines what is meant by an optimal map. It does not, however, indicate how such a map may be found. The method for most efficiently determining an optimal map using a digital computer and the method actually used in a developing biological visual system will clearly differ substantially. This by no means lessens the strength of the prediction of the model. If the model's predictions are correct, then, as a separate question, it is worth considering how biological mechanisms could arrive at the same optimal solution.

\section{Computational complexity}

Before discussing an algorithm for determining the optimal ocular dominance patterns, it is useful to evaluate the difficulty of this task. To understand the complexity of this problem, it helps to describe it in a form that makes explicit the bare essentials of the underlying computational problem.

As noted above in the statement of the model, the LGN and cortex will be modeled by discrete arrays of points. Consider the set of points in the cortex. For a particular distance $\mathbf{D}$, connect all points to neighboring points within a radius $D$. This structure, of points connected by lines, is called a graph (Harary, 1969). The same construction can be done for the LGN layers: in both LGN layers, first connect neighboring (adjacent) points, and then connect corresponding points from the two eyes. This is also a graph. Call these graphs $\mathbf{L}$ (the LGN layers) and $\mathbf{C}$ (the cortex).

For a particular distance $\mathbf{D}$, we are asking the question of whether there is a way of establishing a correspondence between points in $L$ and points in $C$ such that any pair of points connected by a line in $L$ is also connected by a line in $\mathbf{C}$ (i.e., adjacencies are preserved). (If not, then our restriction on $\mathbf{D}$ is too harsh and we could try again with a larger value for D.) This is precisely the subgraph isomorphism problem. This problem bclongs to a class that is called $N P$-complete (Garey and Johnson, 1979). The only known algorithms for solving such problems require time that is exponential in the size of the problem. In our case, the size is proportional to the number of points in the cortical map. In fact, since we want to minimize $\mathbf{D}$ and $\mathbf{N}$, our problem is at least as hard as subgraph isomorphism.

In practical terms, this means that while we can develop an algorithm that tests the model, there is a hard limit on the size of cortical map on which this test can be performed, beyond which the problem becomes intractable. To illustrate the combinatorial nature of this problem, if we allow 60 points in the cortical map, then there are more than $10^{81}$ distinct possibilities for the LGN to cortex mapping-a number roughly equal to the number of particles estimated to be in the universe (Eddington, 1949).

\section{Algorithm}

This scction describes an algorithm that determines, for specified boundary conditions, optimal ocular dominance patterns. Since models of local ocular dominance pattern formation (e.g., von der Malsburg, 1979; Swindale, 1980; Miller et al., 1989) involve starting from random initial conditions, a computer program implementing any one of these models yields different overall patterns each time it is executed. Of course, the claim is that the patterns resulting from these simulations are all similar in character. In contrast, the present algorithm is not a simulation; it will always find the same optimal solution (overall pattern) for a given set of input boundary conditions.

To begin, we construct a discrete representation of the LGN and cortex that embodies the boundary conditions of interest (cat or monkey; Fig. 4). Since the projection from the LGN is proportional and isotropic, in moving from the LGN to the cortex the discrete regions cannot change size, be stretched, or be deformed, and so the resemblance between these points in Figure 4 and black and white billiard balls is not accidentalthey are not arbitrarily malleable. The gray balls represent an empty cortical array, ready to be replaced by black and white balls representing inputs from the LGN. The geniculocortical mapping is thus analogous to picking up the "billiard balls" from the LGN and placing them in some particular arrangement in the cortical array.

We can do better than enumerating every possible arrangement of the LGN points in the cortex by constructing the cortical map incrementally. To construct candidate cortical maps in an orderly manner, first place the foveal point $F$ where it is constrained to lie. Next, choose a point in $\mathbf{L}$ (the LGN layers) adjacent to $F$. The restriction that it must lie within a radius $\mathbf{D}$ restricts where it may be placed in $\mathbf{C}$ (the cortex). To proceed, select one of these possibilities and remember the other possibilities for later. Again, choose a point in $\mathbf{L}$ adjacent to points already mapped, select one of the several possible cortical positions, and defer the others. At some point, we will be unable to proceed because either the map will be complete, or else the current point in $\mathbf{L}$ cannot map to any of the free positions in $\mathbf{C}$ because doing so would involve placing neighbors too far apart (farther than D). If we have found a complete map with all neighbors no farther than $\mathbf{D}$ apart, then we can determine $\mathbf{N}$ and record the solution if it is the best found so far. In both cases, we continue by backtracking to the most recent branch point and selecting the next alternative. The structure of this procedure is thus a tree with branch points whenever an LGN point may map to several possible cortical positions. By continuing until all branches have been explored, the optimal map will be found.

Applying a more sophisticated rule to enforce the adjacency constraints at each branch point can further prune the search tree. Of course, the optimal solution remains unchanged, regardless of the particular algorithm used, because it depends only on the input boundary conditions and the formulation of the model. The choice of algorithm only affects the length of time needed to find this solution. The method employed here is an implementation of a well-known algorithm for subgraph isomorphism (Ullman, 1976). Though still exponential, the resulting search is somewhat reduced from the astronomical figure mentioned earlier.

\section{Results}

From the shape of the LGN layers, the shape of the cortex, and the location of the foveal representation, it is possible to determine, in the manner outlined above, the optimal geniculocortical mapping predicted by the model. The same computer pro- 

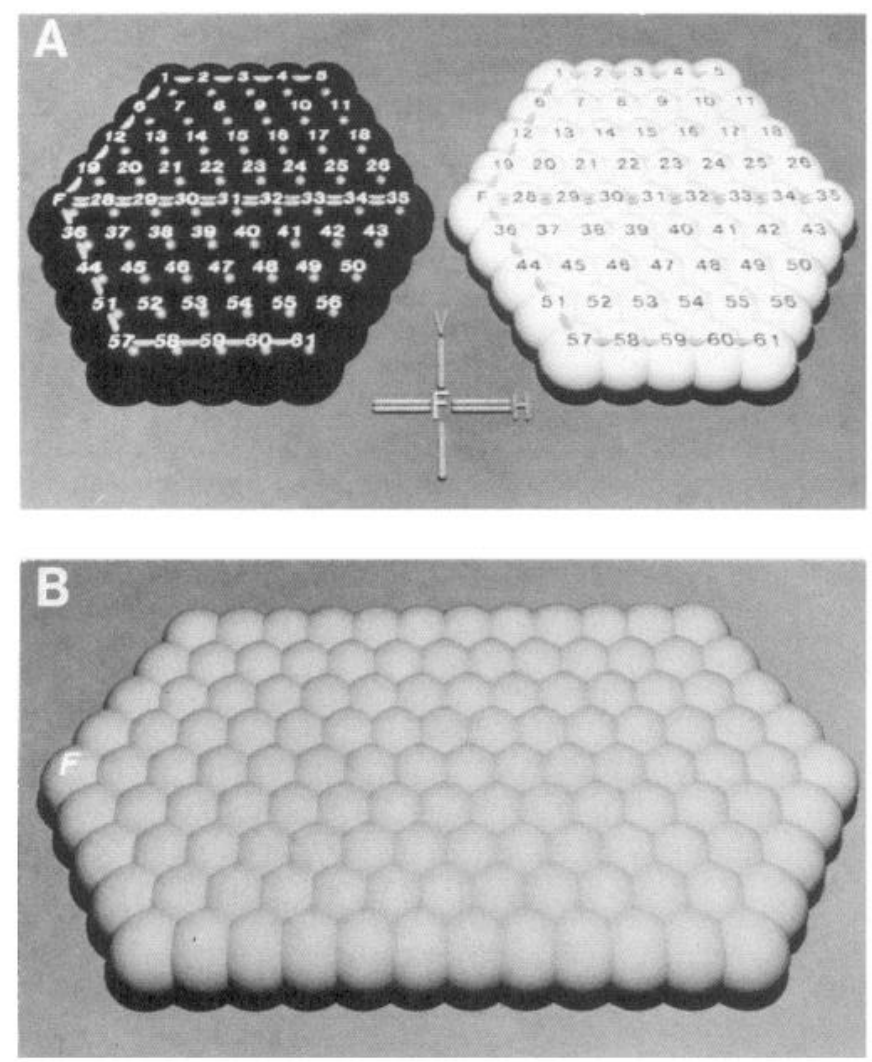

Figure 4. Monkey boundary conditions. The LGN and cortical shapes for the monkey, together with what is taken to be a fixed point in the geniculocortical mapping, the fovea $(F)$, are depicted before the optimal ocular dominance arrangement has been determined. The representative LGN layers $(A)$ for the ipsilateral (black) and contralateral (white) eyes are roughly circular, with the foveal representation $(F)$ lying on the edge, the representation of the horizontal meridian (double line) running across the center, and the vertical meridian (single line) wrapping around the perimeter. The cortical representation $(B)$ is roughly a $2: 1$ ellipse, with the foveal representation $(F)$ mapped to one pole. The gray balls represent the cortical locations to which white or black LGN inputs will be mapped by the model.

gram was used for both the cat and the monkey. The input "boundary conditions" for the monkey are illustrated in Figure 4. The LGN layers representing each eye are roughly circular, and the cortex is a 2:1 ellipse. At the start, the cortical targets are unassigned (gray balls), though the foveal representation will be constrained to lie at the left end of the ellipse. The program considers all possible arrangements of the LGN afferents in the cortical array and, using the criteria of the model, determines the optimal arrangement or mapping - the one that minimizes the cortical distance between LGN neighbors (D) and the number of neighbors that are that distance apart $(\mathbf{N})$. To monitor its progress during execution, the program records each candidate mapping that is an improvement upon its current bound on $\mathbf{D}$ and $\mathbf{N}$. This feature allows us to examine ocular dominance patterns that are almost as good as the optimal one. The determination of optimal mappings for the monkey and cat boundary conditions illustrated in this article takes several CPU-days on a fast computer workstation (e.g., SUN 4/280, 10 MIPs).

The optimal cortical arrangement of the inputs from the two eyes predicted by the model for the monkey is shown in Figure 5 . This pattern of ocular dominance is notably banded, with the predominant orientation of the bands orthogonal to the long axis of the cortex and running perpendicular to much of the border. Some disruption of the orderliness occurs near the foveal representation and at the far periphery. Although it was not an explicit constraint, the resulting cortical visual map is precisely retinotopic. Therefore, the simple, local neighbor-neighbor constraints are sufficient to determine a globally retinotopic map.

The optimal ocular dominance pattern predicted by the model when the cat boundary conditions are used (Fig. 6) is markedly different from that of the monkey. By changing the shape of the LGN to a 2:1 ellipse and moving the area centralis representation to the long side of the cortical ellipse, the optimal arrangement of the two eyes' inputs is much more mottled in appearance. The bands branch irregularly and do not exhibit any one predominant orientation. Note that there is also no general tendency for the ocular dominance bands near the border of striate cortex to be oriented at any particular angle. This stands in sharp contrast with the optimal mapping found for the monkey.

For both the cat and the monkey, these results were reinforced by an examination of ocular dominance patterns that according to the model's criteria were almost as good as the optimal pattern. In each case, these patterns were qualitatively quite similar to the optimal one. For example, none of the near-optimal ocular dominance patterns for the cat exhibited the regular parallel stripes that were characteristic of those found for the monkey.

In summary, changing the shape of the LGN and the placement of the fovea/area centralis but using the same rules for determining the optimal arrangement of ocular dominance results in strikingly different optimal 2-D patterns of ocular dominance. Furthermore, features of the modeled ocular dominance patterns for these two species appear qualitatively similar to the actual biological patterns.

\section{Three-eyed frog}

After the initial presentation of these results (Jones et al., 1988), a particularly interesting biological preparation came to our attention-the three-eyed frog. In the leopard frog (Rana pipiens), axons from only one retina normally form a continuous retinotopic map within the superficial neuropil of the contralateral optic tectum. However, axons from two eyes can be forced to coinnervate one tectal lobe by implanting a third eye primordium into a young embryo (Constantine-Paton and Law, 1978). In this case, retinal ganglion cells project to mutually exclusive eye-specific zones, forming a regular pattern of ocular dominance bands that is consistent from animal to animal and has a characteristic periodicity of $300-400 \mu \mathrm{m}$. During development, retinal growth occurs in concentric annuli, while the optic tectum adds cells only on one end. The retinotectal projection remains plastic, maintaining a functional retinotopic map that shifts across the tectum to compensate for these disparate modes of growth.

This preparation is doubly interesting from the standpoint of our model. Not only does it provide another test case, but since the model depends on the shape of the source and target, and since the shape of the tectum changes as it grows, we can ask if the pattern of ocular dominance changes in a way predicted by the model. Figure 7 shows the ocular dominance bands revealed by injection of HRP into the supernumerary eye. Axons from the other eye project only to the interband regions.

Using tissue kindly provided by M. Constantine-Paton (Department of Biology, Yale University), we measured the dimensions of the tectum of a tadpole (T\&K stage VII) and the dimensions of the tectum of a frog $\left(3 \frac{1}{2}\right.$ weeks postmetamor- 

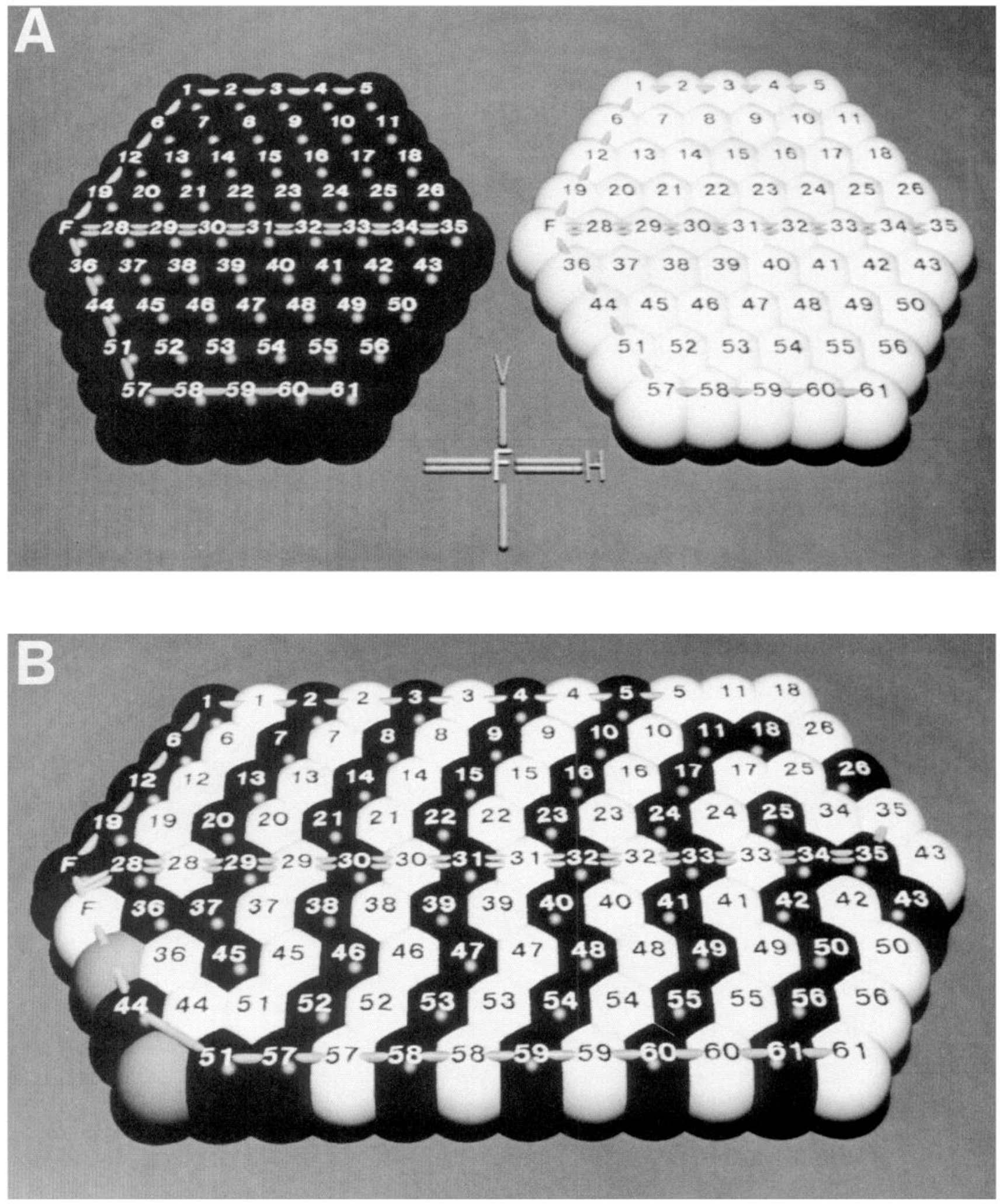

Figure 5. Model prediction for the monkey. Panels show the LGN representations $(A)$ and the optimal cortical ocular dominance pattern (B) predicted by the model for the monkey constraints. The predicted pattern is generally banded, with the bands running roughly perpendicular to the cortical border. The stripelike nature of this pattern is slightly disrupted near the representations of both the fovea $(F)$ and the peripheral visual field (to the right). Taking the width of one ocular dominance column to be 1 unit, the maximum separation between the cortical representations of neighbors is $2.0(\mathbf{D}=2.0)$, and the number of neighbor pairs that far apart is $95(\mathbf{N}=95)$. Same symbols and conventions as Figure 4 . 

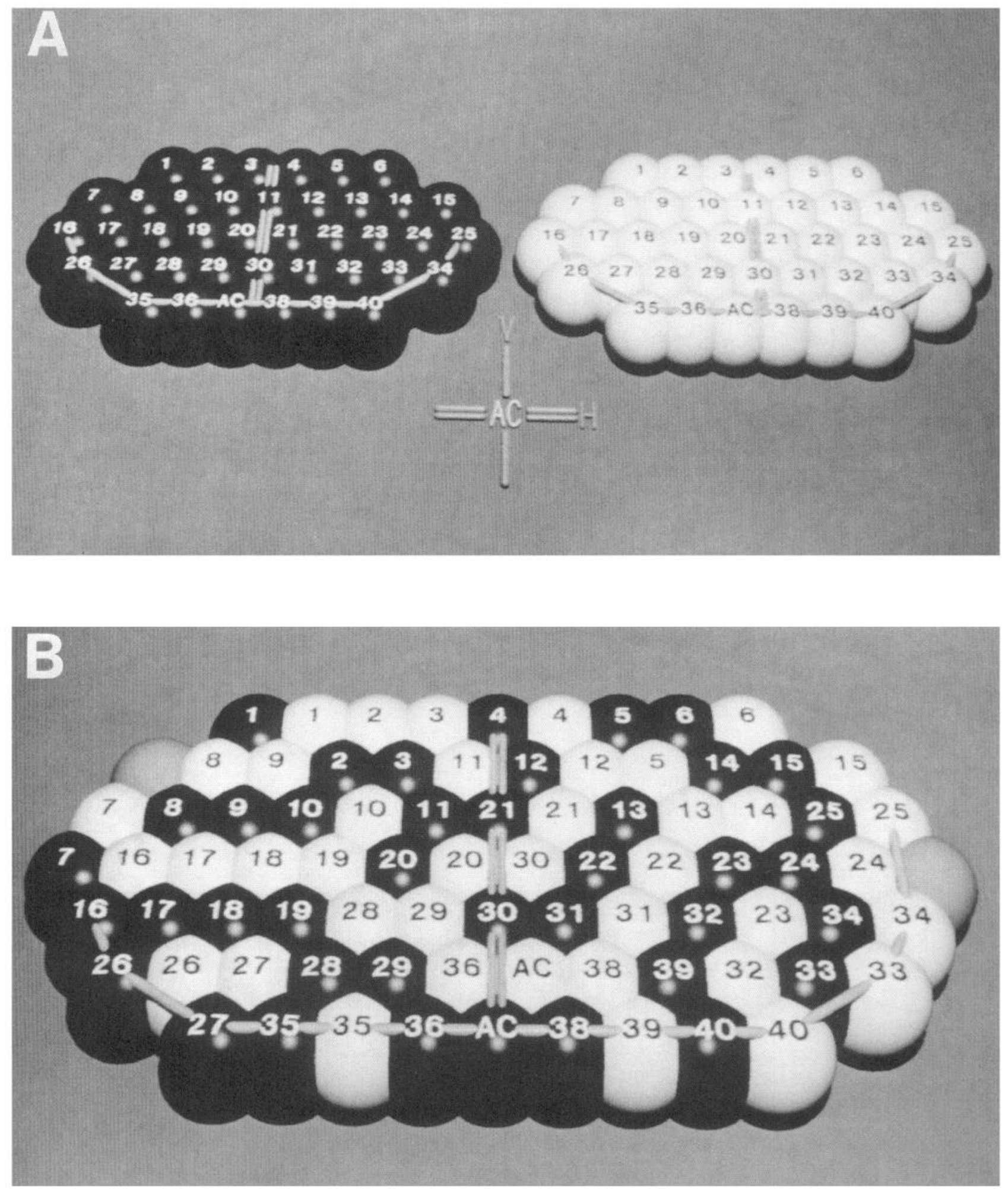

Figure 6. Model prediction for the cat. Panels show the LGN representations $(A)$ and the optimal arrangement of cortical ocular dominance columns $(B)$ predicted by the model for the cat constraints. The predicted ocular dominance pattern for the cat is mottled in appearance, with branching bands that exhibit no predominant orientation. $(D=2.0 ; \mathbf{N}=32)$. Same symbols and conventions as Figures 4 and 5 except $A C$ is area centralis.

phosis). The shape of the retina was assumed to be well approximated by a circular disk. Along with these shapes, our model requires a fixed point in the mapping. A point at the edge of the retina and one pole of the tectum were selected, although the centers of both would have given very similar, if not identical, results. (Whereas an odd choice like mapping the center of the retina to one edge of the tectum could yield considerably different results.) These formed the input boundary conditions 

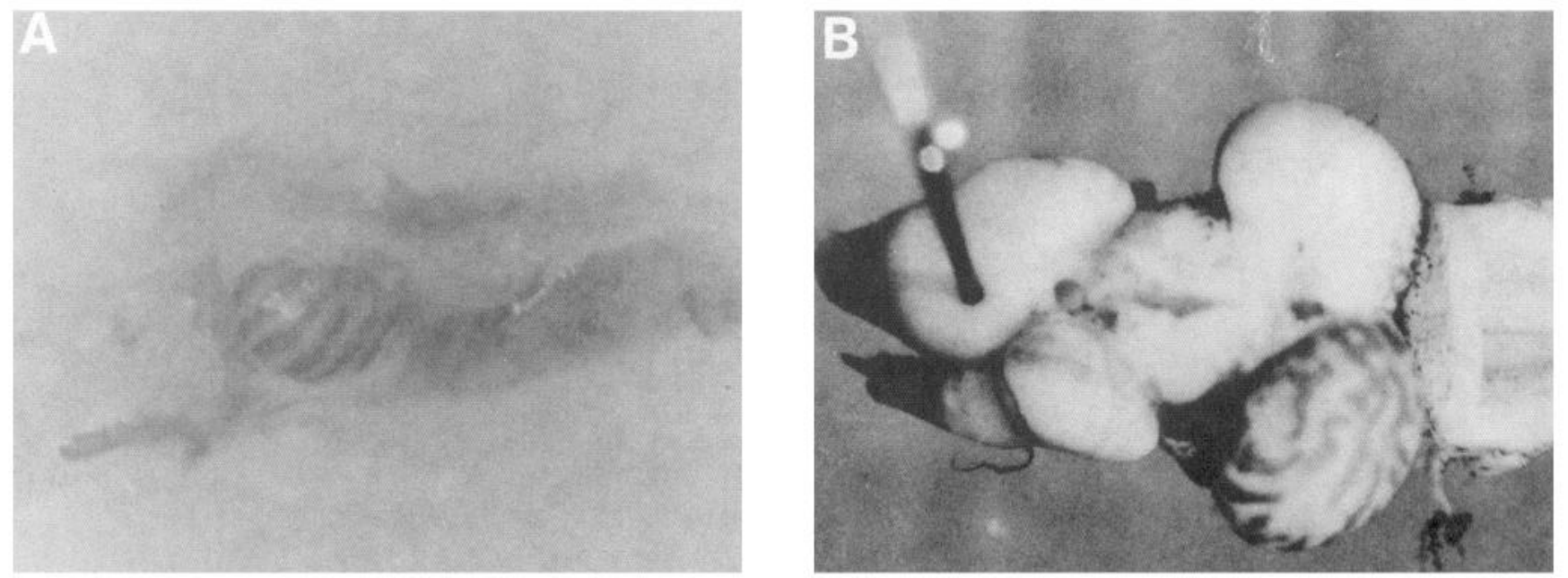

Figure 7. Ocular dominance patterns in three-eyed frogs. A, The pattern of ocular dominance columns in the tectum of the three-eyed tadpole (T\&K stage VII), visualized following injection of HRP into the supernumerary eye. $B$, The pattern of ocular dominance columns in a three-eyed frog $\left(3 \frac{1}{2}\right.$ weeks postmetamorphosis). Magnification is approximately $16 \times$. (Photographs kindly provided by M. Constantine-Paton.)

for the two different ages of three-eyed frog that allowed us to use the same program used for the cat and monkey to determine the optimal ocular dominance patterns predicted by our model.

Figure 8 shows the ocular dominance patterns predicted by the model. The resulting patterns share many qualitative features with the actual patterns observed in three-eyed frogs. For both conditions, the resulting retinotectal projections are retinotopic. For the tadpole, the ocular dominance pattern predicted by the model consists of stereotypically regular parallel stripes. In the postmetamorphic frog, the pattern has somewhat less orderly bands, containing branches and islands. The model thus predicts a change in the ocular dominance pattern with a change in the target shape. It seems clear that the pattern observed in three-eyed frogs changes in a way that is quite similar to that predicted by the model. Comparing Figures 7 and 8 , it is also apparent that, because the number of ocular dominance bands is far fewer than in the cat or monkey, the discretization used to test the model actually comes quite close to the resolution of the three-eyed frog ocular dominance pattern.

\section{Discussion}

\section{Review of the model}

The goal in presenting this model has been to state in a concise yet testable manner the constraints that act upon the overall pattern of ocular dominance. A key idea of the model is that a local "neighborhood" constraint of mapping neighboring locations in the visual field to nearby locations in the cortex acts to impose a map that is globally retinotopic, with left- and righteye inputs interdigitated. Another key idea is that the 2-D shapes of the LGN layers and of primary visual cortex constrain the overall pattern formed by the segregated visual inputs. It is possible to define what is meant by an optimal solution or tradeoff between these constraints. In this sense, an ocular dominance pattern is the solution to a 2-D mapping problem constrained literally by the boundary conditions of the source and target maps.

For different boundary conditions, it is possible to determine the optimal ocular dominance patterns predicted by the model. In particular, this has been done for the cat, monkey, and three- eyed frog. The patterns predicted by the model are qualitatively in quite good agreement with the actual ocular dominance patterns observed.

\section{Possible extensions}

In formalizing the ideas of LeVay et al. (1985) and Anderson et al. (1988), we have centered our attention on the LGN layers and primary visual cortex, in part to avoid coping with the nonuniform distribution of retinal ganglion cells in the retina. It is possible, however, that the present model could be extended to include the retina [as Anderson et al. (1988) did in their original argument] by making use of the results (Schein and de Monasterio, 1987; Schein, 1988; Wässle et al., 1989) that indicate that the ratio of retinal ganglion cells to LGN cells is close to 1:1 throughout most of the visual field. Starting from a detailed knowledge of the density and arrangement of ganglion cells across the retina of the monkey and cat, it is in principle possible to construct an artificial hemiretina. By placing hypothetical "springs" between ganglion cells and their nearest neighbors and allowing the longer springs to contract (and shorter springs to expand), the density would become uniform. It would be interesting to see whether such an exercise would yield different "uniform density" retinal shapes for cat and monkey and, furthermore, whether these shapes would resemble the shapes of the LGN layers.

Both the cat and the monkey are highly binocular animals, with a large part of their visual field seen by both eyes. In the far periphery lie the monocular crescents, each visible only to one eye. Consequently, the LGN layers receiving input from the contralateral eye contain an unpaired monocular region and, in the cortex, there is a thin strip where ocular dominance bands cease to be interdigitated. This region, which receives no input from the ipsilateral eye, constitutes a small fraction of the total area of the striate cortex, as can be seen in Figure 1. The present model could be easily extended to incorporate this feature by adding a thin monocular crescent for one eye in the LGN. The local neighborhood constraints would continue to enforce an orderly retinotopic cortical map, placing the monocular crescent at the edge opposite the foveal representation. The cortical rep- 

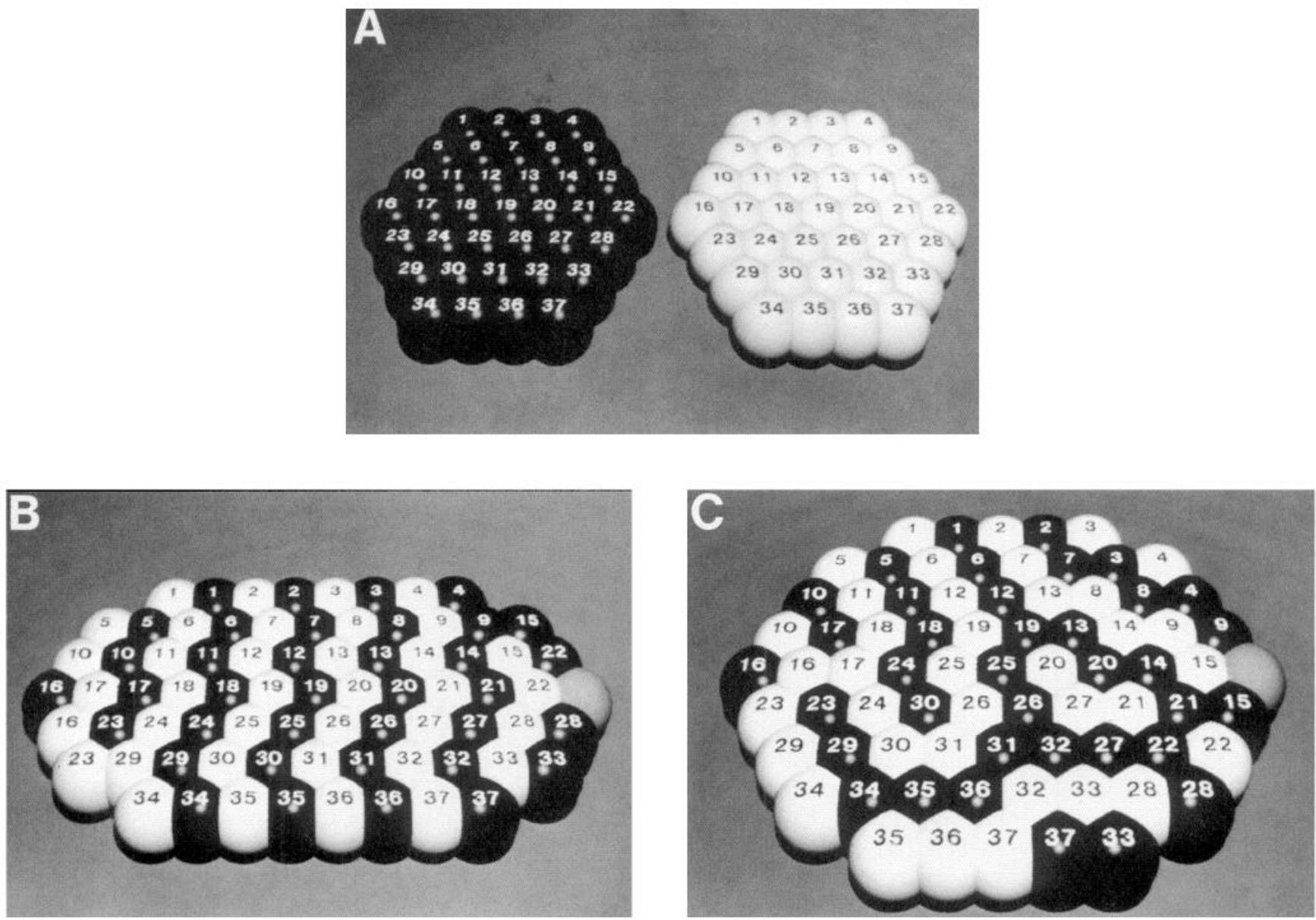

Figure 8. Model predictions for three-eyed frogs. Panels show the shapes of the retinas $(A)$ used by the model to predict the optimal patterns of ocular dominance columns in the tectum of the three-eyed tadpole $(B)$ and postmetamorphic frog $(C)$. The optimal arrangement of ocular dominance columns for the more elliptical tadpole tectum is regularly banded. For the more circular tectum of the older frog, the pattern is less regularly banded, with branches and islands. (tadpole: $\mathbf{D}=2.0, \mathbf{N}=57$; frog: $\mathbf{D}=2.0, \mathbf{N}=28$ ). Same symbols and conventions as Figures 4 and 5 .

resentation of the monocular crescent would not be interspersed with inputs from the other eye, since doing so would unnecessarily separate these inputs from their neighbors.

The model's method for evaluating candidate cortical maps forces the cortical representations for neighboring points in visual space to be close to one another. This is achieved by minimizing the longest cortical distance separating neighbor pairs. Although this rule only applies to neighbor pairs, points that are two or more steps apart are also indirectly constrained to be nearby, though less tightly, by transitivity. For example, two points that are both neighbors of a third point, but not neighbors themselves, will nonetheless be constrained to be relatively close to each other by virtue of both being close to their common neighbor. Other methods for evaluating cortical maps could have been chosen, such as minimizing the sum of the squared distances between neighbors, for example. It is likely that such a rule would predict similar ocular dominance patterns, though it might allow a few long separations between neighbors and, in general, a wider range of separations between neighbors. The rule we have chosen enforces a narrower range of separations and thus a uniformity that could be advantageous when considering local cortical circuitry. It is difficult, however, to imagine any simple rule that could accurately reflect the notion of the biological cost of various possible cortical input arrange- ments. We are confident that the predictions of the model are not artifacts of the particular method chosen for evaluating the cortical maps and that any methods similar in spirit would result in basically the same predictions.

If making predictions from the model for higher-resolution cortical maps were of interest, then as is the common strategy for $N P$-complete problems, there are two avenues of attack. First, it is possible that the rule that forces neighboring LGN points to have nearby cortical representations could be different, yet still similar in spirit to the present one, and that predictions from the revised model would be computationally much more tractable to evaluate. Second, many NP-complete problems yield to reasonably good approximation algorithms, which would not be guaranteed to find the optimal ocular dominance pattern but might be able to find good ones in a reasonable amount of time. Since it is often not clear just how good such solutions are, we chose in this article to limit our attention to an exact algorithm that determines optimal solutions. Now, having taken that step, it would be interesting to see what the model would predict for cortical maps with many more points, perhaps as many as there are cytochrome blobs in the monkey (Hendrickson et al., 1981; Horton and Hubel, 1981; Horton, 1984) or the cat (Murphy et al., 1990), even if it were only an approximation.

While the task of the model is to determine the optimal cor- 
tical ocular dominance patterns and not how these might actually be achieved in a biological system, certain aspects of the model can be cast in a slightly different light that suggests more biologically plausible mechanisms. In order to make the fewest possible assumptions, the model's formulation at least entertains the possibility that each input could map anywhere in the cortex (except the fovea, which is fixed). This could be replaced by a weak prespecification of the overall polarity of the cortical map, perhaps by graded positional markers. Inputs would have an affinity for their retinotopic position as dictated by these gradients but could deviate from that position by some amount. Second, in the model, corresponding points from the two eyes are considered to be neighbors and their cortical representations are thus nearby. This rule could be eliminated and afferents might only be considered neighbors if they were from nearby positions in the same eye, perhaps as indicated by correlated firing patterns. Corresponding points would still map to nearby locations because of transitivity - both would be constrained to be close to a common (retinotopic) cortical location. Computational experiments that we performed, in which LGN afferents were constrained to lie within some radius of their true retinotopic positions, and only inputs from the same eye were considered neighbors, yielded overall ocular dominance patterns similar to those presented here. It is likely that some judiciously selected degree of intrinsic polarity in the cortical map, as well as having local refinements done in parallel, could make the biological "implementation" of this model feasible.

\section{Other models}

Most efforts to date in modeling ocular dominance columns have focused less on the overall patterns observed and more on the developmental process of segregation (e.g., von der Malsburg, 1979; Swindale, 1980; Miller et al., 1989). In these models, segregation results from a compromise between a local attraction or reinforcement involving afferents from the same eye, and a broader repulsion or competition of afferents from different eyes. The constants describing this excitatory-inhibitory interaction determine the width of segregated ocular dominance domains. In some cases, this width can be calculated analytically, or as is more common, it can be discovered from computer simulations.

Simulations by von der Malsburg (1979) demonstrated how the cortex could be a passive site where chemical messengers, carried by afferents, diffuse. It was suggested that several chemical markers could signal an afferent's retinotopic location and eye of origin. Afferents from the two eyes would first be attracted to common cortical locations due to their common retinotopic markers and then be repelled from each other due to their different eye of origin markers. Swindale (1980) showed that cortical interactions between same-eye inputs that are stimulating over short distances, but inhibitory over larger distances, with the reverse being true for interactions between opposite-eye inputs, can yield patterns that resemble ocular dominance patterns. Swindale's model is a general one that did not include detailed assumptions about the underlying cortical physiology, though he pointed out that various specific mechanisms could adequately fulfill these interaction roles. Finally, Miller et al. (1989) described a mechanism that could come into play after LGN afferents have arrived to form an orderly retinotopic cortical map, but with widely overlapping arbors. The extent of these arbors, the degree of correlated activity among afferents, and the extent of lateral interaction in the cortex are the essential parameters of this model.

Though some of Swindale's (1980) simulations modeled small patches of cortex with boundary interactions that led to stripes that were locally perpendicular to the boundary, his other simulations and those of von der Malsburg (1979) and Miller et al. (1989) completely avoided boundary effects by having their small simulated patch of cortex wrap around at the edges to form a torus. In this sense, our own model for the overall pattern and these local models may be viewed as being complementary each addressing different aspects of cortical ocular dominance.

When considering more than one species, like the cat and monkey where the ocular dominance pattern and degree of segregation are different, the parameters of the models described above must be modified to produce the desired results. It is not clear what is gained in moving from an intriguing difference in ocular dominance pattern or degree of segregation to a postulated difference in afferent arbors or cortical interactions. Even among primates, these kinds of models must often employ markedly different parameter values. For example, while the pattern and number of ocular dominance stripes found in humans are quite similar to that seen in the macaque, they are about twice as wide (Horton et al., 1990). Thus, in the Miller et al. (1989) framework, this means cortical interactions must be over a markedly wider scale, or afferent arbors must be markedly broader, or both (K. D. Miller, personal communication).

In a "multiple constraints model" of neural map formation, Fraser and Perkel (1990) present a model that incorporates features from several previous models. The worry with this approach is that at some point a model might become so flexible and have so many parameters that it could fit virtually any data that might be encountered and thus be unfalsifiable. Even evaluating the simpler models poses some difficulties. In each of those mentioned, a variety of parameter settings can yield identical ocular dominance band widths. Diffusion radius and retinotopic specificity both affect the width in von der Malsburg's model. In Swindale's model, the same-eye and opposite-eye interactions can take on a wide variety of forms and still yield ocular dominance stripes of a certain width. Miller and Stryker (1990), citing data mostly from adult cats, comment that according to their model, the width of ocular dominance bands observed in cats (e.g., Anderson et al., 1988) could arise from a variety of intracortical interactions with an excitatory radius as large as $200 \mu \mathrm{m}$, or as small as $50 \mu \mathrm{m}$, or when arbors are the limiting factor, a variety of arbor sizes ranging from uniform arbors with roughly the same diameter as the ocular dominance band spacing, to larger, tapering arbors. The validation of such a model hinges upon the accurate measurement of these biological parameters in the young animal, before ocular dominance segregation begins. As these authors point out, obtaining such measurements will be technically quite difficult.

A recent article by Rojer and Schwartz (1990) illustrates another relevant point, though their effort is not a model in the sense used here. They describe ocular dominance patterns with parameters like width, orientation, and anisotropy. Then, using computer graphics techniques, they are able to recreate their measured patterns by filtering 2-D noise with spatial filters having parameters set to those measured. That the reconstructed patterns would bear a superficial similarity to the original patterns was never in question - the circularity is apparent. With this in mind, the question arises of whether parameters such as afferent arbor diameter and the extent of intracortical interac- 
tions cause the final ocular dominance column width or are a consequence of it. That there is a relationship between adult values of these seems inevitable to us, but it does not establish which determines the other.

\section{Properties of the ocular dominance pattern}

The zebra-stripe pattern of ocular dominance found in the macaque is so striking that certain authors have assumed that it cannot have occurred by chance and instead must have some functional significance. Some have found it a useful exercise to project the cortical ocular dominance pattern back onto the visual field, variously claiming the pattern to be circles, spirals, or horizontal stripes (Hubel and Freeman, 1977; Schwartz, 1977; LeVay et al., 1985). More specifically, it has been suggested that the particular pattern observed has certain desirable properties essential in effective early visual processing (Schein and Lapuerta, 1989). If the basis of the present model and its predictions are valid, then a natural conclusion is that the striped pattern of ocular dominance seen in macaque and human is a simple consequence of constraints that at once provide segregated inputs while keeping representations of neighboring points nearby. The pattern observed in the cat satisfies the same constraints and, given the proximity of inputs from the two eyes, is at no greater disadvantage in early binocular visual processing. In this sense, there may be nothing special about stripes.

LeVay et al. (1985) have also questioned whether there is any special significance to striped ocular dominance patterns. They suggested that the pattern of ocular dominance has more to do with the geography of the cortex than the topography of the visual representation. The intent was to dismiss any functional role of stripes per se and comment on the folded "geography" of macaque striate cortex and local variations in the ocular dominance pattern. However, Horton et al. (1990) found the macaque-like ocular dominance pattern in human primary visual cortex to be quite independent of physical differences, such as the considerable and varied sulcal and gyral geography.

Having suggested that there may not be any special functional significance of striped ocular dominance patterns, a similar criticism may be raised in regard to the interpretation of the fact that the cortical map of the visual field for a variety of species is well approximated by a $\log$ polar conformal mapping function (e.g., Schwartz, 1977). This function is clearly useful in a descriptive sense, though the quality of fit varies for different species and is not accurate in detail (Tootell et al., 1985). While the potential computational benefits of a log polar map have been discussed (e.g., Schwartz, 1980), the possibility remains that the intriguing $\log$ polar nature observed in visual maps simply reflects the optimum packing solution for a high-resolution fovea and a low-resolution periphery (Cavanagh, 1981).

As noted earlier, a consequence of having the visual map isotropic within ocular dominance columns is that whenever these columns systematically line up in stripes, there will necessarily be a $2: 1$ anisotropy introduced into the composite binocular map (Fig. 3). In the cat, the varied orientations of the ocular dominance bands tend to cancel each other out and yield a visual map that is largely isotropic both locally and overall. This is not possible in the macaque, due to the elongated shape of striate cortex. Regardless of the ocular dominance pattern, moving from a circular retinotopic map in the LGN layers to an elliptical map in the striate cortex, an overall anisotropy must be introduced simply because the map is stretched along onc direction. In our framework, two possibilities exist to explain the breakdown of regular stripes near the macaque foveal representation (Fig. 1A). First, it may be crucial for optimal performance foveal vision to have both isotropic monocular inputs as well as an isotropic composite binocular map. Note, however, that when the boundaries of the source and target visual maps are fixed, reducing anisotropy in one part of the target map forces an increased anisotropy in another part. A second possibility is that the more detailed features of the ocular dominance pattern near the foveal representation in the monkey may simply be beyond the resolution at which our model can presently be tested. The accuracy with which the shapes of the LGN and cortex can be represented and the degree of detail within the resulting ocular dominance pattern are limited by the number of points for which it is practical to compute the model's prediction. Interestingly enough, when the resolution at which we have tested our model is comparable to that found in nature, such as for the three-eyed frog, the similarity between the model's predictions and the patterns found in nature is striking.

\section{Developmental considerations}

Drawing upon the results from her work with three-eyed frogs, Constantine-Paton (1983) has proposed that neural map formation is governed by two mechanisms. First, a weaker mechanism, perhaps involving graded chemical markers, matches afferents to destinations by virtue of their positions in the source and target. Second, a stronger mechanism, perhaps being activity dependent, sorts terminals based upon their presynaptic proximity. In this framework, the present model's prespecification of the foveal representation plays the role of the first mechanism and the model's rules for evaluating candidate cortical maps play the role of the second. Though the model is not a developmental one, its value in this regard is in demonstrating how weak, at least in theory, this prespecified mapping mechanism can be. Given only an overall polarity or even just a few fixed points in the mapping, local constraints are sufficient to provide precise retinotopy throughout the cortical map.

The "billiard ball" analogy used in the present model was useful in conveying the concepts of proportionality and local isotropy. It is also suggestive of another aspect of the cortical map-cortical modularity. As further details of the functional organization in primary visual cortex have come to light, Hubel and Wiesel, and others have maintained an overview based on a system of functional cortical modules, each similar, repeated throughout striate cortex (e.g., Hubel and Wiesel, 1979). Recent evidence suggests that at least some aspects of this cortical periodicity could be innate.

In monkeys, the patchy pattern of cytochrome oxidase associated with functional cortical modules is unaffected by the removal of both retinas at such an early time that photoreceptors have not made contact with retinal neurons and cortical layers II and III have not yet formed (Kuljis and Rakic, 1990). In monocularly deprived cats, inputs from the deprived eye are reduced to form isolated beads of ocular dominance, yet are still present in the same number, arrangement, and spacing (Murphy et al., 1989). Since these dramatic changes to the cortical visual inputs leave some aspects of the modularity largely unchanged, the possibility must be entertained that cortical modularity is to a certain extent, if not entirely, innate. Until recently, markers of modularity in cat visual cortex had been limited to labeling the inputs to striate cortex, leaving open the question of whether anything intrinsic to the cortcx was modular, or whether a passive cortex merely reflected a property of its inputs. However, 
cytochrome oxidase, an intrinsic marker, has recently been demonstrated to have a patchy staining pattern in the supragranular layers of cat striate cortex (Murphy et al., 1990), similar to the cytochrome oxidase blobs found in monkeys.

There are some further results suggesting that geniculocortical afferents do not determine the size and spacing of modules in striate cortex. For example, while in humans the area of primary visual cortex is about twice that of the macaque, both have roughly the same number of ocular dominance bands (Horton et al., 1990). Thus, the total number of modules appears to be constant in these two closely related species, despite marked differences in the size of their striate cortex. In the adult cat, clustered horizontal intracortical connections link columns of neurons that respond maximally to visual stimuli of similar orientation (Gilbert and Wiesel, 1983; Ts'o et al., 1986). In kittens, distinctly clustered horizontal connections are apparent as early as the second postnatal week (Luhmann et al., 1986; Callaway and Katz, 1990)-a time when LGN inputs are still diffuse and ocular dominance columns have yet to appear (LeVay et al., 1978). These sorts of results call into question ideas or models that view the cortex as a tabula rasa, an entirely blank and passive target whose modular organization is completely determined by its inputs. Instead, they suggest the alternative that LGN inputs may arrive at a developing cortex that is already to a certain extent modular. If this is the case, local models of ocular dominance segregation could be used to explain how LGN inputs segregate into eye-specific regions, but they could not be used to predict column width, since this would be predetermined by intrinsic cortical factors. It should be noted that, regardless of whether cortex modularity is thought to be determined by intrinsic or extrinsic factors, the present model makes the same predictions for the overall pattern of ocular dominance.

As mentioned previously, it is important not to ascribe any special significance to the difference in the appearance of ocular dominance patterns. In considering developmental mechanisms involved in forming sensory maps in the cortex, it is important to keep in mind the results from the three-eyed frog. Since ocular dominance bands can arise in a species that normally has none, it is wrong to assume that in animals in which they are normally present, ocular dominance columns are due to a specific mechanism. Rather, as already pointed out by Constantine-Paton (Reh and Constantine-Paton, 1985), they may be a natural consequence of a more general mapping mechanism. Combining these two ideas-intrinsic cortical modularity and a general mapping mechanism - it may be that the cortex is not so much organized into ocular dominance columns, as ocular dominance is organized into an already intrinsically columnar cortex. From this viewpoint, the width and spacing of ocular dominance columns may have more to do with the intrinsic modularity of the cortex, and less to do with properties of the cortical inputs themselves.

\section{Appendix}

When considering the ocular dominance pattern observed in the macaque, Le Vay et al. (1985) gave an informal argument along the following lines. A pair of circular disks (each representing one eye's visuotopic map in the LGN) can be sliced into vertical strips and interdigitated to form an ellipse (representing the primary visual cortex) about twice as long as it is wide. In the cat, the shapes of the LGN layers and striate cortex are both roughly $2: 1$ ellipses. Since interdigitating strips stretches the visual map perpendicular to the strips, it may seem at first impossible in the cat to cut the two LGN ellipses into strips and interdigitate them to lie inside a cortical ellipse with the same 2:1 aspect ratio. In fact, if the LGN ellipses are sliced at an angle (in the range of $0^{\circ}$ to $35.3^{\circ}$ ) with the long axis, the resulting strips can be interdigitated to lie in a larger 2:1 ellipse (something the patient reader can verify with paper and scissors). However, the resulting overall visual map in this hypothetical cortex is always considerably skewed, and the foveal representation is displaced from its proper location (in violation of a constraint in our model).

\section{References}

Anderson PA, Olivarria J, Van Sluyters RC (1988) The overall pattern of ocular dominance bands in cat visual cortex. J Neurosci 8:21832200.

Bunt AH, Hendrickson AE, Lund JS, Lund RD, Fuchs AF (1975) Monkey retinal ganglion cells: morphometric analysis and tracing of axonal projections, with a consideration of the peroxidase technique. J Comp Neurol 164:265-286.

Callaway EM, Katz LC (1990) Emergence and refinement of clustered horizontal connections in cat striate cortex. J Neurosci 10:1134-1 153.

Cavanagh P (1981) Size invariance: reply to Schwartz. Perception 10: 469-474.

Clark WE Le Gros (1941) The laminar organization and cell content of the lateral geniculate body in the monkey. J Anat 75:419-433.

Connolly M, Van Essen D (1984) The representation of the visual field in the parvicellular and magnocellular layers of the lateral geniculate nucleus in the macaque monkey. J Comp Neurol 226:544 564

Constantine-Paton M (1983) Position and proximity in the development of maps and stripes. Trends Neurosei 6:32-36.

Constantine-Paton M, Law MI (1978) Eye-specific termination bands in tecta of three-eyed frogs. Science 202:639-641.

Cowcy A (1979) Cortical maps and visual pcrception. The Grindlcy memorial lecture. Q J Exp Psychol 31:1-17.

Cowey A (1981) Why are there so many visual areas? In: The organization of the cerebral cortex (Schmitt FO, Worden FG, Adelman G, Dennis SG, eds), pp 319-413. Cambridge, MA: MIT Press.

Daniel PM, Whitteridge D (1961) The representation of the visual field on the cerebral cortex in monkeys. J Physiol (Lond) 159:203221

Eddington SA (1949) Fundamental theory. London: Cambridge UP.

Fraser SE, Perkel DH (1990) Competitive and positional cues in the patterning of nerve connections. J Neurobiol 21:51-72.

Garey MR, Johnson DS (1979) Computers and intractability. New York: Freeman.

Gilbert CD, Wiesel TN (1983) Clustered intrinsic connections in cat visual cortex. J Neurosci 3:1116-1133.

Harary F (1969) Graph theory. Reading, MA: Addison-Wesley.

Hendrickson AE, Hunt SP, Wu J-Y (1981) Immunocytochemical localization of glutamic acid decarboxylase in monkey striate cortex. Nature 292:605-607.

Horton JC (1984) Cytochrome oxidase patches: a new cytoarchitectonic feature of monkey visual cortex. Philos Trans R Soc Lond [Biol] 304:199-253.

Horton JC, Hubel DH (1981) A regular patchy distribution of cytochrome-oxidase staining in primary visual cortex of macaque monkey. Nature 292:762-764.

Horton JC, Dagi LR, McCrane EP, de Monasterio FM (1990) Arrangement of ocular dominance columns in human visual cortex. Arch Ophthalmol 108:1025-1031.

Hubel DH, Freeman DC (1977) Projection into the visual field of ocular dominance columns in macaque monkey. Brain Res 122:336343.

Hubel DH, Wiesel TN (1965) Binocular interaction in the striate cortex of kittens reared with artificial squint. J Neurophysiol 28:10411059.

Hubel DH, Wiesel TN (1968) Receptive fields and functional architecture of monkey striate cortex. J Physiol (Lond) 195:215-243.

Hubel DH, Wiesel TN (1972) Laminar and columnar distribution of geniculocortical fibers in the macaque monkey. J Comp Neurol 146: $421-450$. 
Hubel DH, Wiesel TN (1977) Functional architecture of macaque monkey visual cortex. Proc R Acad Sci [Biol] 198:1-59.

Hubel DH, Wiesel TN (1979) Brain mechanisms of vision. Sci Am 241(3): 150-162.

Jones DG, Van Sluyters RC, Murphy KM (1988) A computational model for the overall pattern of ocular dominance in striate cortex of the cat and monkey. Invest Ophthalmol Vis Sci [Suppl] 29:297.

Kuljis RO, Rakic P (1990) Hypercolumns in primate visual cortex can develop in the absence of cues from photoreceptors. Proc Natl Acad Sci USA 87:5303-5306.

LeVay S, Hubel DH, Wiesel TN (1975) The pattern of ocular dominance columns in macaque visual cortex revealed by a reduced silver stain. J Comp Neurol 179:223-244.

LeVay S, Stryker MP, Shatz CJ (1978) Ocular dominance columns and their development in layer IV of the cat's visual cortex: a quantitative study. J Comp Neurol 179:223-244.

LeVay S, Wiesel TN, Hubel DH (1980) The development of ocular dominance columns in normal and visually deprived monkeys. $J$ Comp Neurol 191:1-51.

LeVay S, Connolly M, Houde J, Van Essen DC (1985) The complete pattern of ocular dominance stripes in the striate cortex and visual field of the macaque monkey. J Neurosci 5:486-501.

Luhmann HJ, Martínez Millán L, Singer W (1986) Development of horizontal intrinsic connection in cat striate cortex. Exp Brain Res 63:443-448.

Miller KD, Stryker MP (1990) The development of ocular dominance columns: mechanisms and models. In: Connectionist modeling of brain function: the developing interface (Hanson SJ, Olson CR, eds), pp 255-305. Cambridge, MA: MIT Press.

Miller KD, Keller JB, Stryker MP (1989) Ocular dominance column development: analysis and simulation. Science 245:605-615.

Murphy KM, Van Sluyters RC, Jones DG (1989) Analysis of the overall pattern of ocular dominance in normal and visually deprived cats. Invest Ophthalmol Vis Sci [Suppl] 30:30.

Murphy KM, Van Sluylers RC, Jones DG (1990) Cytochrome-oxidase activity in cat visual cortex: is it periodic? Soc Neurosci Abstr 16: 292.

Reh TA, Constantine-Paton M (1985) Eye-specific segregation requires neural activity in three-eyed Rana pipiens. J Neurosci 5:11321143.

Rojer AS, Schwartz EL (1990) Cat and monkey cortical columnar patterns modeled by bandpass-filtered 2-D white noise. Biol Cybern 62:381-391.

Sanderson KJ (1971) The projection of the visual field to the lateral geniculate and medial interlaminar nuclei in the cat. J Comp Neurol 143:101-118.

Schein SJ (1988) Anatomy of macaque fovea and spatial densities of neurons in foveal representation. J Comp Neurol 269:479-505.

Schein SJ, de Monasterio FM (1987) Mapping of the retinal and geniculate neurons onto striate cortex of macaque. J Neurosci 7:9961009.

Schein SJ, Lapuerta P (1989) Primary visual cortex in macaque is a conformal map of its true sensory surface, a composite of the two retinas. Soc Neurosci Abstr 15:1106.

Schwartz EL (1977) Afferent geometry in the primate visual cortex and the generation of neuronal trigger features. Biol Cybern 28:1-14.

Schwartz EL (1980) Computational anatomy and functional architecture of striate cortex: a spatial mapping approach to perceptual coding. Vision Res 20:645-669.

Schwartz EL (1985) On the mathematical structure of the visuotopic mapping of macaque striate cortex. Science 227:1065-1066.

Stone J (1965) A quantitative analysis of the distribution of ganglion cells in the cat's retina. J Comp Neurol 124:337-352.

Swindale NV (1980) A model for the formation of ocular dominance stripes. Proc R Soc Lond [Biol] 208:243-264.

Tootell RBH, Silverman MS, Switkes E, De Valois RD (1985) On the mathematical structure of the visuotopic mapping of macaque striate cortex. Science 227:1065-1066.

Tootell RBH, Switkes E, Silverman MS, Hamilton SL (1988) Functional anatomy of macaque striate cortex. II. Retinotopic organization. J Neurosci 8:1531-1568.

Ts'o DY, Gilbert CD, Wiesel TN (1986) Relationships between horizontal interactions and functional architecture in cat striate cortex as revealed by cross-correlation analysis. J Neurosci 6:1160-1170.

Tusa RJ, Palmer LA, Rosenquist AC (1978) The retinotopic organization of area 17 (striate cortex) in the cat. J Comp Neurol 117:213235.

Ullman JR (1976) An algorithm for subgraph isomorphism. J ACM 23:32-42.

von der Malsburg C (1979) Development of ocularity domains and growth behavior of axon terminals. Biol Cybern 32:243-264.

Wässle H, Grünert U, Röhrenbeck J, Boycott BB (1989) Cortical magnification factor and the ganglion cell density of the primate retina. Nature 341:643-646.

Wässle H, Grünert U, Röhrenbeck J, Boycott BB (1990) Retinal ganglion cell density and cortical magnification factor in the primate Vision Res 30:1897-1911. 